\title{
Quasi-homogeneous black hole thermodynamics
}

\author{
Hernando Quevedo ${ }^{1,2,3, a}$, María N. Quevedo ${ }^{4, b}$, Alberto Sánchez ${ }^{5, c}$ \\ ${ }^{1}$ Instituto de Ciencias Nucleares, Universidad Nacional Autónoma de México, AP 70543, Ciudad de México 04510, Mexico \\ 2 Dipartimento di Fisica and ICRA, Università di Roma "Sapienza", 00185 Rome, Italy \\ ${ }^{3}$ Institute of Experimental and Theoretical Physics, Al-Farabi Kazakh National University, Almaty 050040, Kazakhstan \\ ${ }^{4}$ Departamento de Matemáticas, Universidad Militar Nueva Granada, Cra 11 No. 101-80, Bogotá, DE, Colombia \\ ${ }^{5}$ Departamento de posgrado, CIIDET, AP752, Querétaro, QRO 76000, Mexico
}

Received: 26 December 2018 / Accepted: 4 March 2019 / Published online: 13 March 2019

(C) The Author(s) 2019

\begin{abstract}
Although the fundamental equations of ordinary thermodynamic systems are known to correspond to firstdegree homogeneous functions, in the case of non-ordinary systems like black holes the corresponding fundamental equations are not homogeneous. We present several arguments, indicating that black holes should be described by means of quasi-homogeneous functions of degree different from one. In particular, we show that imposing the firstdegree condition leads to contradictory results in thermodynamics and geometrothermodynamics of black holes. As a consequence, we show that in generalized gravity theories the coupling constants like the cosmological constant, the Born-Infeld parameter or the Gauss-Bonnet constant must be considered as thermodynamic variables.
\end{abstract}

\section{Introduction}

An important property of thermodynamic laboratory systems is that their fundamental equations are given in terms of homogeneous functions of first degree. Recall that a fundamental equation is a function that relates an extensive thermodynamic potential (entropy or energy) with the extensive thermodynamic variables necessary to describe the system. Then, the homogeneity condition is a consequence of the fact that extensive variables are additive [1]. Generalizations of the extensivity property have been also considered in the literature and concepts like sub-extensive and supra-extensive variables have been introduced to correctly describe the behavior of certain thermodynamic systems. Recently in [2], we proposed to classify thermodynamic systems into ordinary and non-ordinary by using an exact math-

\footnotetext{
a e-mail: quevedo@nucleares.unam.mx

b e-mail: maria.quevedo@unimilitar.edu.co

c e-mail: asanchez@ciidet.edu.mx
}

ematical concept, namely, the concept of homogeneous and generalized homogeneous functions.

Let $\Phi$ denote a fundamental thermodynamic potential [3] which could be either the entropy or the internal energy. Let $\left\{E^{a}\right\}(a=1, \ldots, n)$ denote the set of extensive variables that are necessary to describe a thermodynamic system with $n$ degrees of freedom. Then, a system described by the fundamental equation $\Phi\left(E^{a}\right)$ is called ordinary if $\Phi$ is a homogeneous function

$\Phi\left(\lambda E^{a}\right)=\lambda^{\beta} \Phi\left(E^{a}\right)$,

where $\lambda$ is a real constant and $\beta>0$ is the degree of homogeneity. In general, ordinary systems are characterized by the value $\beta=1$. If $\Phi$ is a generalized homogeneous function, i.e., [4]

$\Phi\left(\lambda^{\beta_{1}} E^{1}, \ldots, \lambda^{\beta_{n}} E^{n}\right)=\lambda^{\beta_{\Phi}} \Phi\left(E^{1}, \ldots, E^{n}\right)$,

where $\beta_{a}=\left(\beta_{1}, \ldots, \beta_{n}\right)$ are real constants, and $\beta_{\Phi}$ is the degree of homogeneity, the system is called non-ordinary. In the literature, generalized homogeneous functions are also known as quasi-homogeneous functions; in fact, the idea of considering quasi-homogenous thermodynamics in several contexts, including black hole physics and geometric representations of thermodynamics, has been analyzed previously by Belgiorno and Cacciatore in a series of publications [57]. Accordingly, non-ordinary and quasi-homogeneous are terms that can be used indistinctly to refer to thermodynamic systems that are not described by homogeneous functions of first degree.

As pointed out in [5-7], black holes and other nonordinary systems should be considered as quasi-homogeneous systems for different reasons and, in fact, it is a consequence of the well known non-extensivity property of certain thermodynamic systems. In particular, quasi-homogeneity is important to correctly describe the behavior of non-ordinary systems near the critical points [5]. In a different context, by 
investigating the thermodynamics of AdS black holes [8], it has been long suggested $[9,10]$ that the cosmological constant can be considered as a thermodynamic variable. It turns out that in this case the cosmological constant can naturally be thought of as a pressure and the mass of the black hole as the enthalpy of the spacetime. By following this idea, it has been recently established that AdS black holes can be investigated from the point of view of chemistry and that there exists an intriguing physical analogy between van der Waals fluids and black holes [11]. This is a remarkable result that deserves further investigation and will certainly contribute to the understanding of the physical properties of black holes. It is therefore important to find out why the cosmological constant can be assumed to be an additional thermodynamic variable for black holes. In this work, we will see that this assumption is not only possible, but also necessary in order for black holes to be quasi-homogeneous systems. Although black hole thermodynamics is perfectly well defined without cosmological constant, the recently proposed extended thermodynamics with cosmological constant reveals new physical aspects of black holes, hitherto unseen.

On the other hand, differential geometric methods have been applied during the past few decades in classical thermodynamics to investigate the stability and the critical points of thermodynamic systems [12-15]. The main goal of these studies consists in finding connections between the thermodynamic properties of the system and the geometric properties of the corresponding equilibrium space. The approach of thermodynamic geometry consists in introducing a completely fixed Riemannian metric into the space of equilibrium states of a given system. In this case, the components of the metric are associated with the second moment of the fluctuations of a particular thermodynamic potential [14]. A second approach consists in demanding that the metric of the equilibrium space be invariant with respect to Legendre transformations, i.e., with respect to the choice of thermodynamic potential. This approach is known as geometrothermodynamics (GTD) [15]. In this case, the metric of the equilibrium space is derived by using Legendre invariance and turns out to contain certain degree of arbitrariness. We will see below that this arbitrariness becomes completely fixed as a consequence of imposing the quasi-homogeneity condition.

Thus, in this work, we will explore the consequences of demanding quasi-homogeneity for black holes in two different contexts. First, we will see how the quasi-homogeneity condition fixes the thermodynamic metric which is used in GTD to describe black holes and, moreover, that GTD is able to detect the non-correct use of this condition. Second, we explore black hole thermodynamics from the point of view of quasi-homogeneity and show that it dictates the thermodynamic properties of the parameters that enter the fundamental equation of black holes. If quasi-homogeneity is not handled correctly, it turns out that the thermodynamic properties of a black hole configuration can change drastically.

This work is organized as follows. In Sect. 2, we review the main physical consequences of imposing homogeneity in ordinary thermodynamic systems. In Sect. 3, we explore thermodynamic quasi-homogeneity in the context of GTD, and show that systems with intrinsic thermodynamic interaction can lead to contradictory results for the corresponding equilibrium space, when the quasi-homogeneity condition is not implemented properly. In Sect. 4, we analyze the fundamental thermodynamic equation of black hole configurations in several gravity theories, and show that the physical parameters, such as the coupling constants, that enter the action in a field theoretical approach must be considered as thermodynamic variables as a consequence of the quasi-homogeneity condition. Moreover, we show the thermodynamic inconsistencies that can arise when the quasi-homogeneity condition is not applied appropriately. Finally, in Sect. 5, we review our results, and propose some tasks for future investigations.

\section{Homogeneity of ordinary systems}

Given a thermodynamic system through its fundamental equation $\Phi=\Phi\left(E^{a}\right)$, one defines the corresponding intensive variables as

$I_{a}=\frac{\partial \Phi}{\partial E^{a}}$,

so that the first law of thermodynamics is simply

$d \Phi=I_{a} d E^{a}$.

These relations are valid in general for homogeneous and quasi-homogeneous systems, and are well-defined if the fundamental potential $\Phi$ is differentiable, a condition which is usually assumed in classical thermodynamics.

Ordinary or homogeneous thermodynamic systems can be characterized by the degree of homogeneity $\beta$, which is determined through the condition (1). This condition is also known in the thermodynamic literature as the static scaling hypothesis [16]. In the case of ordinary laboratory systems with $\beta=1$, the intensive variables $I_{a}$ are homogeneous functions of degree zero, i.e., $I_{a}\left(\lambda E^{a}\right)=I_{a}\left(E^{a}\right)$, a property which is in accordance with our intuitive idea of intensive quantities since they do not depend on the size of the system [1]. Ordinary laboratory systems have also the property that their fundamental potentials can be inverted. Indeed, the homogeneity, continuity, differentiability and monotonic property of the entropy $S$ imply that it can be inverted with respect to the energy $U$ which is, in turn, a homogeneous function of first degree [1]. For concreteness, let us consider as a particular example the simple case of an ideal gas with 
a fixed number of particles $N$, whose fundamental equation is given by [1]

$S(U, V, N)=k_{B} N\left(\ln \frac{V}{N}+\frac{3}{2} \ln \frac{U}{N}\right)$,

where $k_{B}$ is the Boltzmann constant and $V$ is the volume of the gas. This is a first-degree homogeneous function, i.e, $S(\lambda U, \lambda V, \lambda N)=\lambda S(U, V, N)$ which can be inverted with respect to $U$ and yields

$U(S, V, N)=N e^{\frac{2 S}{3 k_{B} N}}\left(\frac{V}{N}\right)^{-2 / 3}$,

with $U(\lambda S, \lambda U, \lambda N)=\lambda U(S, V, N)$. We see that in this case a change of representation preserves the homogeneity property.

In the case of Legendre potentials, i.e., thermodynamic potentials that are obtained from the fundamental potentials by means of Legendre transformations, the situation is completely different. Consider, for instance, the Legendre potentials of the ideal gas

$$
\begin{aligned}
F(T, V, N)= & U-T S=\frac{3}{2} k_{B} N T \\
& \times\left\{1-\ln \left[\frac{3}{2} k_{B} T\left(\frac{V}{N}\right)^{2 / 3}\right]\right\}, \\
H(S, P, N)= & U+P V=\frac{9 N P^{2}}{4} e^{-\frac{2 S}{3 k_{B} N}} \\
& -\frac{8 N}{27 P^{2}} e^{\frac{2 S}{k_{B} N}}, \\
G(T, P, N)= & U-T S+P V=\frac{3}{2} N k_{B} T \\
& \times\left[1-\ln \left(\frac{3 P^{2}}{2 k_{B} T}\right)\right]-\frac{4 N}{9 k_{B} T},
\end{aligned}
$$

which can all be written explicitly in terms of the corresponding variables. None of these potentials can be considered as a homogeneous function. However, if we rescale the extensive variables only, we obtain $F(T, \lambda V, \lambda N)=\lambda F(T, V, N)$ and similar relations for the remaining potentials. This implies that Legendre potentials preserve the homogeneity property only at the level of the extensive variables. Intense variables do not rescale as a consequence of their zero degree of homogeneity.

For a general value of $\beta \neq 1$, the situation is completely different. First, the intensive variables are not represented by homogeneous functions of zero degree. Instead, their degree can be set as $\beta-1$ so that it is positive for supra-extensive variables and negative for sub-extensive parameters. Also, a fundamental potential cannot be inverted in general, implying that a particular representation must be chosen to perform the physical investigation of the system properties. Moreover, the constant $\beta$ enters explicitly the Euler and Gibbs-Duhem identities (summation over repeated indices) [2],

$I_{a} E^{a}=\beta \Phi, \quad(1-\beta) I_{a} d E^{a}+E^{a} d I_{a}=0$,

respectively, which relate extensive and intensive variables. This implies that homogeneous systems with $\beta \neq 1$ will behave differently from a thermodynamic point of view.

In the case of quasi-homogeneous systems, defined through the condition (2), the situation is similar. The fundamental potentials cannot be inverted in general and the Euler and Gibbs-Duhem identities become [2]

$\beta_{a b} I^{a} E^{b}=\beta_{\Phi} \Phi, \quad\left(\beta_{a b}-\beta_{\Phi} \delta_{a b}\right) I^{a} d E^{b}+\beta_{a b} E^{b} d I^{a}=0$,

with $I_{a}=\delta_{a b} I^{b}$ and

$\delta_{a b}=\operatorname{diag}(1, \ldots, 1), \quad \beta_{a b}=\operatorname{diag}\left(\beta_{1}, \ldots, \beta_{n}\right)$.

The diagonal matrix $\beta_{a b}$ contains all the information about the quasi-homogeneity of the extensive variables. The corresponding intensive variables are in general not given as homogeneous functions of zero degree, implying that, in fact, they may depend on the size of the system. It is, therefore, necessary to handle them with care, always taking into account their non-trivial degree of quasi-homogeneity.

\section{Quasi-homogeneity in geometrothermodynamics}

To describe thermodynamics from a geometric point of view, essentially two different methods have been used so far. The approach of thermodynamic geometry assumes that the equilibrium space $\mathcal{E}$ is geometrically described by a Hessian metric

$g^{H}=\Phi_{, a b} d E^{a} d E^{b}=\frac{\partial^{2} \Phi}{\partial E^{a} \partial E^{b}} d E^{a} d E^{b}$,

where $\Phi=\Phi\left(E^{a}\right)(a=1, \ldots, n)$ represents the fundamental equation of the thermodynamic system under consideration. In the energy representation $\Phi=U$, the corresponding metric is known as the Weinhold metric [13] whereas if the thermodynamic potential is chosen as minus the entropy $\Phi=-S$, the Ruppeiner metric is obtained [14]. In general, however, it is possible to use as potential for the Hessian metric any thermodynamic potential that can be obtained from $U$ or $S$ by means of a Legendre transformation [17].

The second approach of GTD is based upon the use of Legendre invariance, i.e., the property that classical thermodynamics does not depend on the choice of thermodynamic potential [15]. To consider Legendre invariance as an invariance with respect to coordinate transformations, it is necessary to introduce the auxiliary phase space $\mathcal{T}$, in which all the thermodynamic variables $\left\{\Phi, E^{a}, I^{a}\right\}$ are considered as independent coordinates. Then, the space $\mathcal{T}$ is endowed with 
a Legendre invariant Riemannian metric $G$ and a canonical contact 1-form $\Theta=d \Phi-I_{a} d E^{a}$. Whereas the contact 1 -form is uniquely defined modulo a conformal function, there are three classes of Legendre invariant metrics [18, 19], namely,

$$
G^{I / I I}=\left(d \Phi-I_{a} d E^{a}\right)^{2}+\left(\xi_{a b} E^{a} I^{b}\right)\left(\chi_{c d} d E^{c} d I^{d}\right),
$$

which are invariant under total Legendre transformations. Here $\xi_{a b}$ and $\chi_{a b}$ are diagonal constant $(n \times n)$-matrices. For $\chi_{a b}=\delta_{a b}=\operatorname{diag}(1, \ldots, 1)$, the resulting metric $G^{I}$ can be used to investigate systems with at least one first-order phase transition. Alternatively, for $\chi_{a b}=\eta_{a b}=\operatorname{diag}(-1, \ldots, 1)$, we obtain the metric $G^{I I}$ which has been used to describe systems with second-order phase transitions. The third class

$$
G^{I I I}=\left(d \Phi-I_{a} d E^{a}\right)^{2}+\left(E_{a} I_{a}\right)^{2 k+1} d E^{a} d I^{a}, \quad k \in \mathbb{Z},
$$

is invariant with respect to partial Legendre transformations and is used to describe ordinary systems.

In GTD, the equilibrium space $\mathcal{E}$, with the set of coordinates $\left\{E^{a}\right\}$, is considered as a subspace of the phase space $\mathcal{T}$ and is defined by the embedding map $\varphi: \mathcal{E} \rightarrow \mathcal{T}$ with $\varphi:\left\{E^{a}\right\} \mapsto\left\{\Phi\left(E^{a}\right), E^{a}, I^{a}\left(E^{a}\right)\right\}$ and $\varphi^{*}(\Theta)=0$. Then, any metric $G$ in $\mathcal{T}$ induces a metric $g$ in $\mathcal{E}$ by means of the pullback $g=\varphi^{*}(G)$. This means that in GTD there can be also three different classes of metrics $g^{I}, g^{I I}$ and $g^{I I I}$ for the equilibrium space.

Quasi-homogeneity plays now an important role in the determination of the final form of $g^{I}$ and $g^{I I}$. Indeed, black hole configurations, which according to our previous description are a particular case of quasi-homogeneous systems, are also characterized by certain dependence on the statistical ensemble chosen for their description [20], indicating that they cannot be completely independent of the choice of thermodynamic potential. This implies that quasi-homogeneous systems can be invariant only with respect to total Legendre transformations and, consequently, they can be described by the metrics $g^{I}$ and $g^{I I}$, only. This is in accordance with our previous results obtained in GTD in which we use only the metric $g^{I I}$ to describe black hole systems with second-order phase transitions. Moreover, by using the Euler-identity for quasi-homogeneous systems in the derivation of the metrics of $\mathcal{E}$, we obtain [2]

$$
g^{I / I I}=\beta_{\Phi} \Phi \xi_{a}^{c} \Phi_{b c} d E^{a} d E^{b}=\beta_{\Phi} \Phi \xi_{a}^{c} \frac{\partial^{2} \Phi}{\partial E^{b} \partial E^{c}} d E^{a} d E^{b},
$$

where $\xi_{a}{ }^{c}=\delta_{a}{ }^{c}=\operatorname{diag}(1, \ldots, 1)$ for $g^{I}$ and $\xi_{a}{ }^{c}=\eta_{a}{ }^{c}=$ $\operatorname{diag}(-1,1, \ldots, 1)$ for $g^{I I}$. We then conclude that quasihomogeneous systems must be described in GTD by a particular set of metrics which is invariant with respect to total
Legendre transformations. Notice that the multiplicative constant $\beta_{\Phi}$ in front of the metrics $g^{I / I}$ corresponds exactly to the arbitrary constant that remains free in the analysis of quasi-homogeneous fundamental equations. This means that in GTD this arbitrariness leads to a simple conformal factor which does not affect the geometric properties of the equilibrium space.

We now illustrate in a particular example the importance of correctly handling the homogeneity properties of the fundamental equations. Consider the Reissner-Nordström black hole in any dimension. Their corresponding line element is given as in Eq. (50) with [21] (from now on, we will use geometric units with $G=c=\hbar=k_{B}=1$ )

$$
f(r)=1-\frac{16 \pi M}{(d-2) \omega_{(d-2)}} \frac{1}{r^{d-3}}+\frac{8 \pi}{(d-2)(d-3)} \frac{Q^{2}}{r^{2(d-3)}},
$$

where $\omega_{(d-2)}=2 \pi^{\frac{d-1}{2}} / \Gamma\left(\frac{d-1}{2}\right)$. The outer event horizon is, therefore, given by

$$
\frac{2(d-2)}{\omega_{(d-2)}} r_{+}{ }^{(d-3)}=M+M \sqrt{1-\frac{d-2}{2(d-3)} \frac{Q^{2}}{M^{2}}} .
$$

On the other hand, the entropy in $d$ dimensions can be computed by using the formula $[21,22]$,

$S=\left[\frac{2(d-2)}{\omega_{(d-2)}}\right]^{\frac{(d-2)}{(d-3)}} r_{+}(d-2)$

Then, from Eqs. (18) and (19) we obtain explicitly the entropy function

$S(M, Q)=\left(M+M \sqrt{1-\frac{d-2}{2(d-3)} \frac{Q^{2}}{M^{2}}}\right)^{\frac{d-2}{d-3}}$,

which represents the fundamental thermodynamic equation of the Reissner-Nordström black hole in $d$ dimensions. It is easy to see that it corresponds to a non-ordinary system because the degree of homogeneity is $\frac{d-2}{d-3}$. In this particular case, the fundamental equation can be inverted, yielding in the mass representation the equation

$M(S, Q)=\frac{1}{2} S^{\frac{d-3}{d-2}}+\frac{d-2}{4(d-3)} \frac{Q^{2}}{S^{\frac{d-3}{d-2}}}$,

which satisfies the first law [23]

$d M=T d S+\phi d Q$

where $T$ is the temperature and $\phi$ the electric potential. From the fundamental equation and the first law it is, therefore, possible to derive the complete set of thermodynamic variables of the system. Analogously, in GTD all the geometric information about the equilibrium space can be obtained from the 


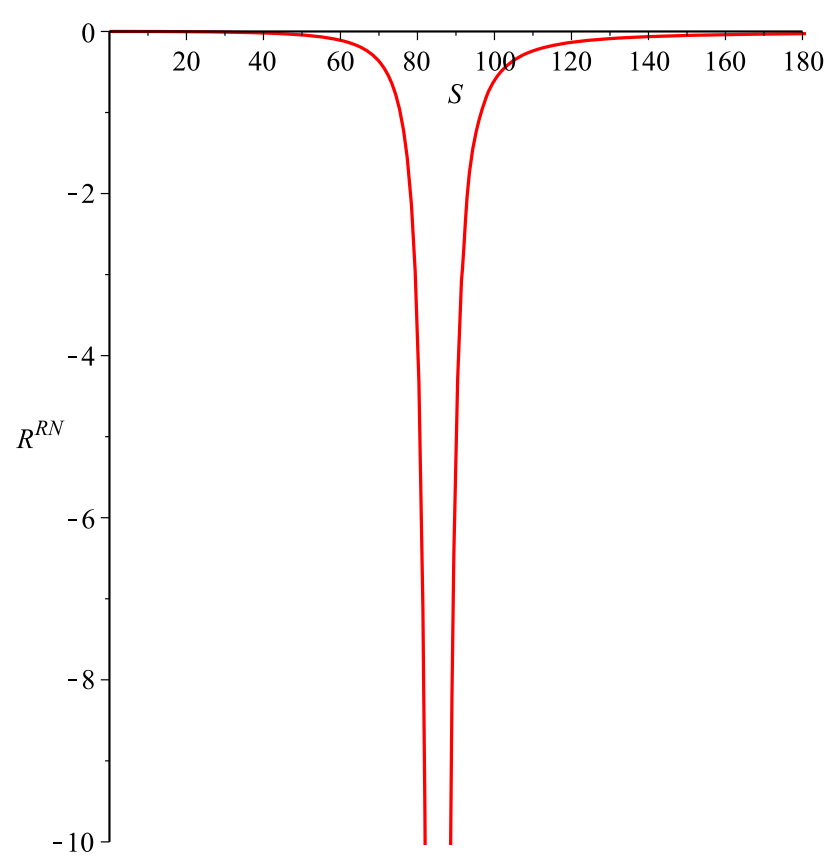

Fig. 1 The curvature scalar $R^{R N}$ as a function of the entropy $S$ with $Q=10$ and $d=5$

fundamental equation. Indeed, the metric $g^{I I}$ with $\Phi=M$ and $E^{a}=\{S, Q\}$ leads to

$g^{I I}=\beta_{M} M\left(-\frac{\partial^{2} M}{\partial S^{2}} d S^{2}+\frac{\partial^{2} M}{\partial Q^{2}} d Q^{2}\right)$,

which for the fundamental equation (21) can be expressed as

$$
\begin{aligned}
g^{R N}= & \beta_{M} \frac{M}{2(d-3)(d-2)}\left\{\frac { ( d - 3 ) } { S ^ { 2 } } \left[S^{\frac{2(d-3)}{(d-2)}}\right.\right. \\
& \left.\left.+\frac{(2 d-5)}{2} Q^{2}\right] d S^{2}+(d-2)^{2} d Q^{2}\right\} .
\end{aligned}
$$

A straightforward computation shows that the corresponding scalar curvature is

$$
R^{R N}=\frac{8(d-2)(d-3)^{2} S^{\frac{2(d-3)}{(d-2)}}}{\beta_{M} M\left[2(d-3) S^{\frac{2(d-3)}{(d-2)}}-\left(2 d^{2}-9 d+10\right) Q^{2}\right]^{2}} .
$$

The non-zero curvature indicates that this system is characterized by the presence of a non-trivial thermodynamic interaction. Moreover, second-order phase transitions are determined by the curvature singularities $\left(R^{R N} \rightarrow \infty\right)$, which are present at those points where $2(d-3) S^{\frac{2(d-3)}{(d-2)}}-\left(2 d^{2}-\right.$ $9 d+10) Q^{2}=0$. This condition has non-trivial solutions as illustrated in a particular case in Fig. 1.

According to the results presented in Sect. 4, the degree of homogeneity remains free and, in principle, can be fixed arbitrarily. In [23], it was suggested that in the case of black holes it can be fixed to 1 . This would imply that for a certain choice of thermodynamic variables, black holes can be considered as ordinary systems. We will see now that in GTD this assumption can lead to contradictory results. In fact, the Reissner-Nordström fundamental equation (21) in the new variables

$m=M^{\frac{d-2}{d-3}}, \quad q=Q^{\frac{d-2}{d-3}}$,

reduces to

$m(S, q)=\left[\frac{1}{2} S^{\frac{d-3}{d-2}}+\frac{2-d}{2(d-3)} \frac{q^{\frac{2(d-3)}{d-2}}}{S^{\frac{d-3}{d-2}}}\right]^{\frac{d-2}{d-3}}$,

which is a homogenous function of degree 1 . In this case, according to Eq. (16), the metric $g^{I I}$ for $\Phi=m$ and $E^{a}=$ $\{S, q\}$ reduces to

$g^{I I}=\beta_{m} m\left(-\frac{\partial^{2} m}{\partial S^{2}} d S^{2}+\frac{\partial^{2} m}{\partial q^{2}} d q^{2}\right)$.

Using the Reissner-Nordström fundamental equation (27) in the new variables, we obtain

$g^{R N}=\beta_{m} F(S, q)\left(\frac{d S^{2}}{S^{2}}-\frac{d q^{2}}{q^{2}}\right)$,

with

$F(S, q)=\frac{m^{2} q^{\frac{2 d}{d-2}} S^{\frac{6}{d-2}}\left[S^{\frac{2(d-3)}{(d-2)}}\left(d^{2}-7 d+12\right)-q^{\frac{2(d-3)}{(d-2)}}\left(d^{2}-4 d+4\right)\right]}{S^{\frac{2(d-3)}{(d-2)}}(d-3)-q^{\frac{2(d-3)}{(d-2)}}(d-2)}$.

The computation of the thermodynamic curvature of this metric shows that it vanishes identically. According to GTD, this means that this system has no intrinsic thermodynamic interaction, which contradicts the result obtained above with the original variables $M$ and $Q$. We conclude that in GTD it is not allowed to perform a transformation of variables at the level of the fundamental equation with the aim of describing a black hole configuration by means of a homogeneous function of first degree. GTD detects such transformations by changing the geometric properties of the equilibrium space.

\section{Quasi-homogeneity in black hole thermodynamics}

Ordinary systems are characterized by entropies that depend on the volume of the system. This is not the case of black holes. Indeed, 4D black hole thermodynamics is based upon the Bekenstein-Hawking relation $S=A / 4$ that relates the entropy of the black hole $S$ with its horizon area $A$. This is the first fact that indicates a non-standard thermodynamic behavior in black holes. The horizon area, in turn, is a geometric quantity that can be calculated by using the metric of the corresponding spacetime and depends on the physical parameters of the black hole. In the case of the EinsteinMaxwell theory, the most general black hole is described by 
the Kerr-Newman spacetime which contains only three independent parameters, namely, the mass $M$, angular momentum $J$ and electric charge $Q$. A straightforward computation of the horizon area leads to the fundamental equation [23]

$S(M, J, Q)=\pi\left(2 M^{2}-Q^{2}+2 \sqrt{M^{4}-J^{2}-M^{2} Q^{2}}\right)$,

that according to the postulates of black hole thermodynamics should satisfy the first law

$d S=\frac{1}{T} d M-\frac{\Psi}{T} d Q-\frac{\Omega}{T} d J$,

where $T, \Psi$ and $\Omega$ are the corresponding intensive variables, which are interpreted as the temperature, electric potential and angular velocity at the horizon, respectively. Then, we obtain

$$
\begin{aligned}
T & =\frac{1}{2 M S} \sqrt{M^{4}-J^{2}-M^{2} Q^{2}}, \\
\Psi & =\frac{\pi Q}{M S}\left(M^{2}+\sqrt{M^{4}-J^{2}-M^{2} Q^{2}}\right), \\
\Omega & =\frac{\pi J}{M S} .
\end{aligned}
$$

The rescaling $M \rightarrow \lambda^{\beta_{M}} M, J \rightarrow \lambda^{\beta_{J}} J$ and $Q \rightarrow \lambda^{\beta_{Q}} Q$ shows that if the conditions

$\beta_{M}=\frac{1}{2} \beta_{S}, \quad \beta_{J}=\beta_{S}, \quad \beta_{Q}=\frac{1}{2} \beta_{S}$,

are satisfied, the function (31) is quasi-homogeneous of degree $\beta_{S}$, i.e., $S\left(\lambda^{\beta_{S} / 2} M, \lambda^{\beta_{S}} J, \lambda^{\beta_{S} / 2} Q\right)=\lambda^{\beta_{S}} S(M, J, Q)$. Moreover, it is then easy to show that the only intensive variable with zero degree of quasi-homogeneity is $\Psi$, whereas $T$ and $\Omega$ are of degree $-\beta_{S} / 2$. In particular, the Hawking temperature $T$ will not behave as the temperature of an ordinary system. Since the constant $\beta_{S}$ remains arbitrary, one is tempted to fix it by introducing new thermodynamic variables. In fact, this is possible because the degree of any quasihomogeneous function can always be set equal to one by choosing the variables appropriately [4]. For instance, the change of variables

$S \rightarrow s^{2}, \quad J \rightarrow j^{2}, \quad M \rightarrow m, Q \rightarrow q$,

transforms the fundamental equation (31) into

$s(m, j, q)=\pi^{1 / 2}\left(2 m^{2}-q^{2}+2 \sqrt{m^{4}-j^{4}-m^{2} q^{2}}\right)^{1 / 2}$,

which is a first-degree function. If this equation were to describe a thermodynamic system, it must satisfy in particular the first law of thermodynamics

$d s=\frac{1}{t} d m-\frac{\psi}{t} d q-\frac{\omega}{t} d j$, from which we obtain the corresponding intensive variables

$$
\begin{aligned}
t & ==\frac{1}{m s} \sqrt{m^{4}-j^{4}-m^{2} q^{2}}, \\
\psi & =\frac{\pi q}{m s^{2}}\left(m^{2}+\sqrt{m^{4}-j^{4}-m^{2} q^{2}}\right), \\
\omega & =\frac{2 \pi j^{3}}{m s^{2}} .
\end{aligned}
$$

All these quantities have zero degree of homogeneity and as such can be considered as genuine intensive variables. Some minor differences appear in the behavior of these intensive variables as functions of the extensive variables when compared with the intensive variables $T, \Psi$ and $\Omega$ that follow from the quasi-homogeneous fundamental equation (31). This is illustrated in Fig. 1.

However, if we consider the corresponding heat capacities

$$
\begin{aligned}
C_{Q, J}^{K N} & =T\left(\frac{\partial T}{\partial S}\right)_{Q, J}=\frac{4 M^{3} S^{2} T}{M^{4}+J^{2}-4 M^{3} S T}, \\
c_{q, j} & =t\left(\frac{\partial t}{\partial s}\right)_{q, j} \\
& =\frac{1}{\pi} \frac{m^{3} s^{4} t}{q^{2}\left(m^{4}-j^{4}\right)+4 m^{2} j^{4}+m s t\left(m^{2} q^{2}+2 j^{4}\right)},
\end{aligned}
$$

major differences appear. In Fig. 2, we illustrate the behavior of these capacities as functions of the entropies. We see that the differences are crucial. The quasi-homogeneous heat capacity $C$ shows clearly a second-order phase transition which is lacking in the analysis of the capacity $c$. This shows that a change of thermodynamic variables in order to get a first degree homogeneous functions can drastically change the thermodynamic properties of the system (Fig. 3).

This simple example shows the importance of correctly handling the homogeneous or quasi-homogeneous character of fundamental equations. In the next subsections, we will present several examples that illustrate the way we propose to handle quasi-homogeneous systems.

\subsection{Einstein-Maxwell gravity with cosmological constant}

In the Einstein-Maxwell theory with cosmological constant $\Lambda$, which follows from the action

$\mathcal{S}=\frac{1}{16 \pi} \int d^{4} x \sqrt{-g}\left(R-F_{\mu \nu} F^{\mu \nu}-2 \Lambda\right)$,

the most general solution representing a black hole configuration is known as the Kerr-Newman-AdS solution [24]. It contains four independent parameters, namely, the mass $m$, specific angular momentum $a=j / m$, electric charge $q$ and cosmological constant $\Lambda$. Since these parameters are usually defined for asymptotically flat metrics, in the case of asymptotically AdS spacetimes the problem appears that several 

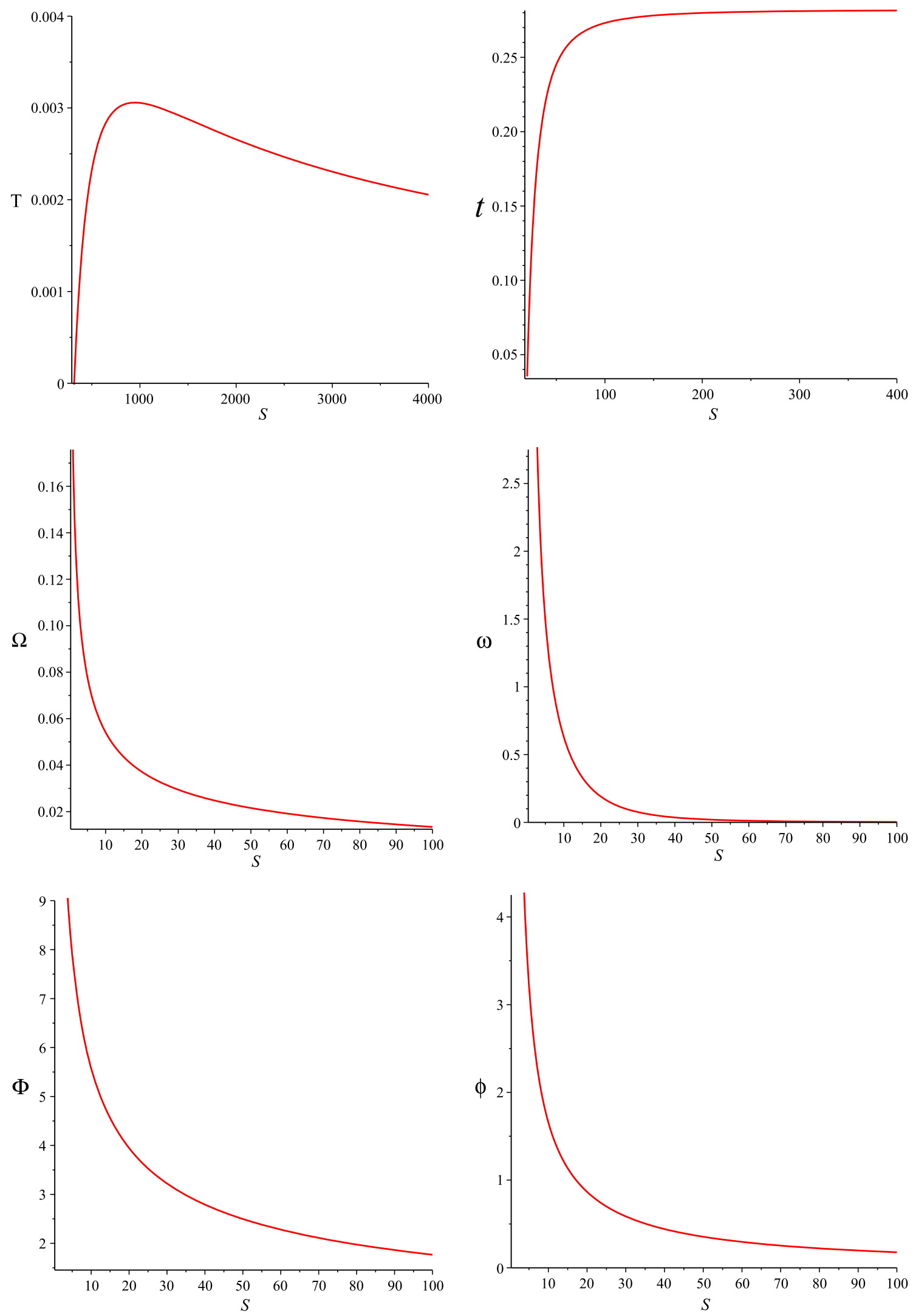

Fig. 2 Behavior of the intensive quasi-homogeneous $(T, \Omega, \Phi)$ and homogeneized $(t, \omega, \phi)$ as functions of the quasi-homogeneous entropy $S$ and the homogeneized entropy $s$ for the particular values $J=j=5$ and $Q=q=10$ 

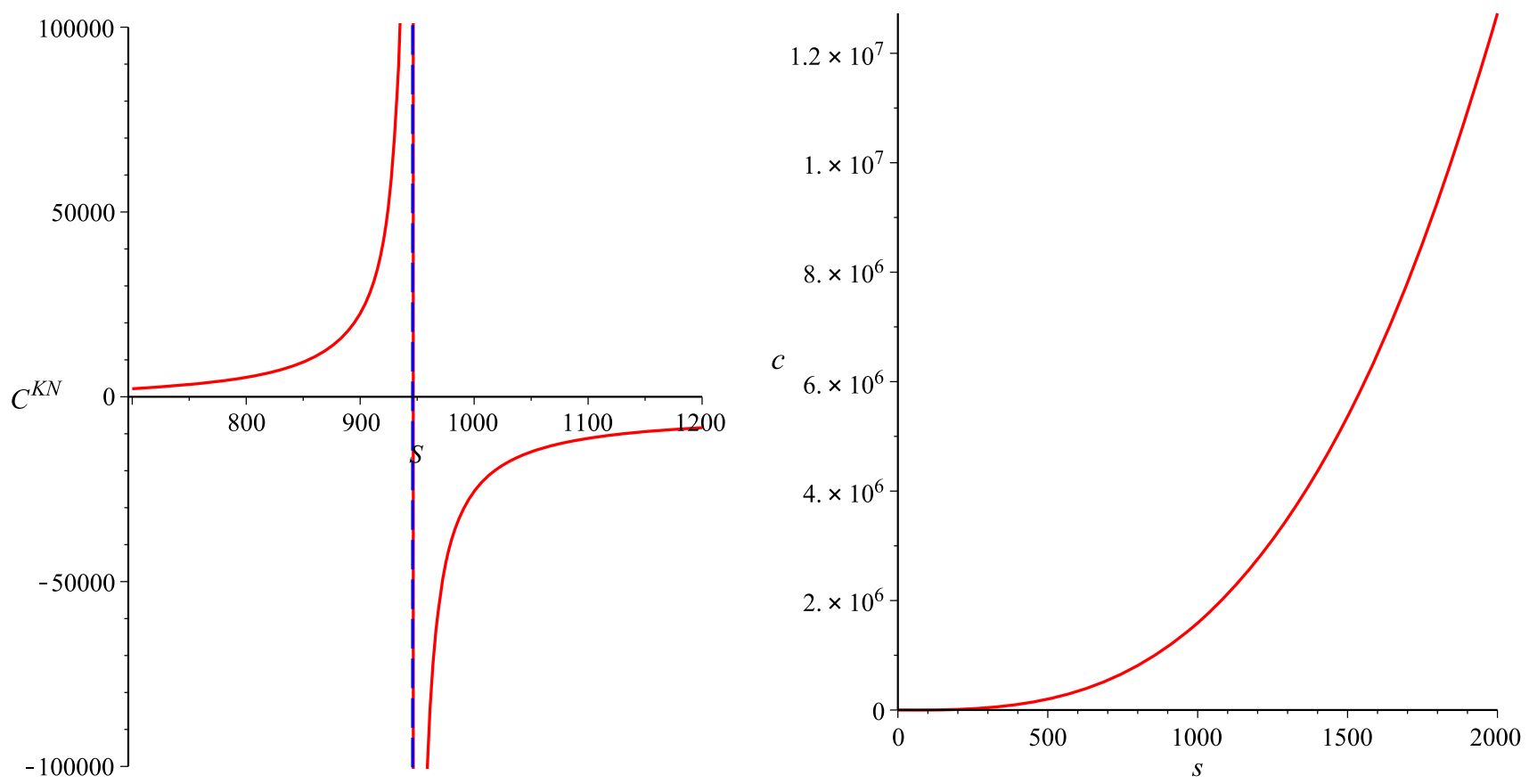

Fig. 3 Heat capacity $C$ (left) and heat capacity $c$ (right) as functions of the entropy $S$ and entropy $s$, respectively, with $J=j=5$ and $Q=q=10$

definitions are possible. In particular, the physical angular velocity can be defined as the difference between the angular velocity at infinity and on the horizon [8]. In the context of the formalism of isolated horizons [25,26], it is also possible to address this problem and it has been shown that the intrinsic physical parameters in this case are given by [27]

$M=\frac{m}{\Xi^{2}}, \quad J=\frac{a m}{\Xi^{2}}, \quad Q=\frac{q}{\Xi}, \quad \Xi=1+\frac{\Lambda}{3} \frac{J^{2}}{M^{2}}$.

These parameters are related to the physical entropy $S$ by means of the Smarr formula

$M^{2}=J^{2}\left(-\frac{\Lambda}{3}+\frac{\pi}{S}\right)+\frac{S^{3}}{4 \pi^{3}}\left(-\frac{\Lambda}{3}+\frac{\pi}{S}+\frac{\pi^{2} Q^{2}}{S^{2}}\right)^{2}$

which is equivalent to the fundamental thermodynamic equation, relating the total mass (energy) $M$ of the black hole with the extensive variables $S, Q$, and $J$. It is easy to see that this equation cannot be inverted; this is one of the first signals indicating that it corresponds to a non-ordinary system.

Performing the rescaling of the extensive variables $M \rightarrow$ $\lambda^{\beta_{M}} M, S \rightarrow \lambda^{\beta_{S}} S, J \rightarrow \lambda^{\beta_{J}} J, Q \rightarrow \lambda^{\beta_{Q}} Q$, it is easy to see that the function (47) does not satisfy either the homogeneity nor the quasi-homogeneity condition. However, if we consider the cosmological constant $\Lambda$ as a thermodynamic variable which rescales as $\Lambda \rightarrow \lambda^{\beta_{\Lambda}} \Lambda$, the fundamental equation (47) turns out to be a quasi-homogeneous function if the conditions

$\beta_{J}=\beta_{S}, \beta_{\Lambda}=-\beta_{S}, \beta_{Q}=\frac{1}{2} \beta_{S}, \beta_{M}=\frac{1}{2} \beta_{S}$

are fulfilled. This means that the degree of the function is defined modulo the coefficient $\beta_{S}$.

Although the cosmological constant is originally not interpreted as a thermodynamic variable, we see that if the KerrNewman-AdS black hole is to be considered as a quasihomogeneous system, then the requirement appears that the cosmological constant must be a thermodynamic variable. Although the coefficient $\beta_{S}$ remains arbitrary, one can consider it as positive to take into account the sub or supra extensive character of the entropy of the entropy. It then follows that $\Lambda$ should be interpreted as an intensive variable. In fact, by using a completely different approach, it was shown that $\Lambda$ can be interpreted as the pressure of the system [9-11].

\subsection{Einstein-Born-Infeld gravity}

Consider the Einstein-Born-Infeld action in $3+1$ dimensions, which is given by the expression [28]

$$
\begin{aligned}
\mathcal{S}= & \int \mathcal{L} \sqrt{-g} d^{4} x, \quad \mathcal{L}=\frac{1}{16 \pi}(R-2 \Lambda) \\
& +\frac{b^{2}}{4 \pi}\left(1-\sqrt{1+\frac{2 F}{b^{2}}}\right) .
\end{aligned}
$$


Here, $F$ is the electromagnetic invariant defined as $F=$ $\frac{1}{4} F_{\mu \nu} F^{\mu \nu}$, and $b$ is known as the Born-Infeld parameter, which in string theory is related to the string tension $\alpha^{\prime}$ as $b=\frac{1}{2 \pi \alpha^{\prime}}$.

A particular spherically symmetric solution of the corresponding field equations is described by the line element

$d s^{2}=-f(r) d t^{2}+f^{-1}(r) d r^{2}+r^{2} d \Omega_{(d-2)}^{2}$,

where $d \Omega^{2}{ }_{(d-2)}$ the line element on the $(d-2)$ - dimensional unit sphere ( $d=4$ in this case) and

$$
\begin{aligned}
f(r)= & 1-\frac{2 M}{r}-\frac{\Lambda}{3} r^{2}+\frac{2 b^{2} r^{2}}{3} \\
& \times\left(1-\sqrt{1+\frac{Q^{2}}{b^{2} r^{4}}}\right)+\frac{4 Q^{2}}{3 r^{2}} \mathcal{F}_{1} .
\end{aligned}
$$

Here $\mathcal{F}_{1}$ represents the hypergeometric function

$$
\mathcal{F}_{1}=\mathcal{F}\left(\frac{1}{4}, \frac{1}{2}, \frac{5}{4},-\frac{Q^{2} \pi^{2}}{b^{2} S^{2}}\right),
$$

$M$ is the ADM mass and $Q$ the electric charge. The horizons of this $3+1$ dimensional black hole are determined by the roots of the lapse function $f(r)$. In terms of the outer horizon radius $r_{+}$and the electric charge $Q$, the black hole mass is given by $[28,29]$

$$
\begin{aligned}
M\left(r_{+}, Q\right)= & \frac{r_{+}}{2}-\frac{\Lambda}{6} r_{+}^{3}+\frac{b^{2} r_{+}^{3}}{3} \\
& \times\left(1-\sqrt{1+\frac{Q^{2}}{b^{2} r_{+}^{4}}}\right)+\frac{2 Q^{2}}{3 r_{+}} \mathcal{F}_{1} .
\end{aligned}
$$

In four dimensions, the fundamental equation that relates the entropy of the black hole with the horizon area leads to $S=\pi r_{+}^{2}$ for spherically symmetric black holes. Then, the mass of the black hole becomes

$$
\begin{aligned}
& M(S, Q)=\frac{1}{2} S^{1 / 2}+S^{3 / 2} \\
& \times\left[-\frac{\Lambda}{6}+\frac{b^{2}}{3}\left(1-\sqrt{1+\frac{Q^{2}}{b^{2} S^{2}}}\right)\right]+\frac{2 Q^{2}}{3 \sqrt{S}} \mathcal{F}_{1},
\end{aligned}
$$

where for the sake of simplicity we have normalized the entropy as $S \rightarrow \pi S$. This relation represents the fundamental equation for the Born-Infeld-AdS black hole presented above.

We now analyze the rescaling properties of the fundamental equation (54). Since the only independent variables are $S$ and $Q$, we perform the transformation $S \rightarrow \lambda^{\beta_{S}} S$ and $Q \rightarrow \lambda^{\beta} Q Q$. Then, the resulting function does not satisfy the quasi-homogeneous condition. However, if we also perform the transformations $b \rightarrow \lambda^{\beta_{b}} b$ and $\Lambda \rightarrow \lambda^{\beta_{\Lambda}} \Lambda$, then the fundamental equation (54) becomes quasi-homogeneous $M \rightarrow \lambda^{\beta_{M}} M$ under the conditions
$\beta_{Q}=\frac{1}{2} \beta_{S}, \quad \beta_{\Lambda}=-\beta_{S}, \quad \beta_{b}=-\frac{1}{2} \beta_{S}, \quad \beta_{M}=\frac{1}{2} \beta_{S}$.

All the coefficients of quasi-homogeneity are determined in terms of the degree $\beta_{S}$, which remains arbitrary as a consequence of the definition of the quasi-homogeneous functions. The above results show that imposing the quasi-homogeneity condition for this black hole implies that the cosmological constant and the Born-Infeld parameter as well must be considered as thermodynamic variables.

\subsection{Einstein-Maxwell-Gauss-Bonnet gravity}

The particular case of the Einstein-Maxwell-Gauss-Bonnet (EMGB) gravity in $4+1$ dimensions can be obtained by adding the Gauss-Bonnet invariant and a matter Lagrangian to the Einstein-Hilbert action, i.e.,

$$
\begin{aligned}
\mathcal{S}= & \kappa \int d^{5} x \sqrt{-g}\left[R+\alpha\left(R^{2}-4 R^{\mu \nu} R_{\mu \nu}\right.\right. \\
& \left.\left.+R^{\alpha \beta \gamma \delta} R_{\alpha \beta \gamma \delta}\right)-2 \Lambda+F_{\alpha \beta} F^{\alpha \beta}\right],
\end{aligned}
$$

where $\kappa$ is related to the Newton constant, and $\alpha$ is the GaussBonnet coupling constant.

A five dimensional spherically symmetric solution of this theory can be explicitly written by using the line element (50) with $d=5$ and the metric function $[30,31]$

$$
f(r)=1+\frac{r^{2}}{4 \alpha}-\frac{r^{2}}{4 \alpha} \sqrt{1+\frac{8 \alpha M}{r^{4}}-\frac{8 \alpha Q^{2}}{3 r^{6}}+\frac{4 \alpha \Lambda}{3}} .
$$

The two parameters $M$ and $Q$ are identified as the mass and electric charge of the system. The above solution describes an asymptotically anti-de-Sitter black hole only if the expression inside the square root is positive and the function $f\left(r_{H}\right)=0$ on the horizon radius, i. e.,

$$
\begin{aligned}
1 & +\frac{8 \alpha M}{r_{H}^{4}}-\frac{8 \alpha Q^{2}}{3 r_{H}^{6}}+\frac{4 \alpha \Lambda}{3} \\
& >0, \quad \frac{\Lambda}{3} r_{H}^{6}-2 r_{H}^{4}+2(M-2 \alpha) r_{H}^{2}-\frac{2}{3} Q^{2}=0 .
\end{aligned}
$$

By choosing the units appropriately, the Bekenstein-Hawking entropy in five dimensions can be written as $S=r_{H}^{3}$. Then, assuming the validity of the Bekenstein-Hawking formula in this case, the corresponding thermodynamic fundamental equation in the mass representation becomes

$M=2 \alpha+S^{2 / 3}+\frac{Q^{2}}{3 S^{2 / 3}}-\frac{\Lambda}{6} S^{4 / 3}$.

Notice that to guarantee the positiveness of the mass in general, we must choose $\alpha>$ and $\Lambda<0$.

Following the procedure described above, it can be shown that the fundamental equation (59) turns out to be a quasihomogeneous function only if we transform all the variables 
as $S \rightarrow \lambda^{\beta_{S}} S, Q \rightarrow \lambda^{\beta_{Q}} Q, \alpha \rightarrow \lambda^{\beta_{\alpha}} \alpha$, and $\Lambda \rightarrow \lambda^{\beta_{\Lambda}} \Lambda$. Moreover, the following relationships between the coefficients must be fulfilled

$\beta_{Q}=\beta_{M}=\beta_{\alpha}=\frac{2}{3} \beta_{S}, \quad \beta_{\Lambda}=-\frac{2}{3} \beta_{S}$.

In this case, the cosmological constant and the Gauss-Bonnet constant $\alpha$ turn out to be thermodynamic variables in order to preserve the quasi-homogeneity properties of the black hole configuration.

It should be mentioned that here we assumed the validity of the Bekenstein-Hawking entropy formula in EMGB gravity. However, it is known that in higher dimensional theories the Wald entropy formula should be applied, which does not necessarily coincides with the Bekenstein-Hawking formula. In fact, this is the case of EMGB gravity for which it was found that [32]

$S_{W}=S_{B H}\left(1+\frac{12 \alpha}{r_{H}^{2}}\right)$.

We can now solve this cubic algebraic equation, express the radius $r_{H}$ in terms of $S_{W}$ and replace the resulting expression in Eq. (58) to obtain the corresponding fundamental equation. The resulting equation cannot be written in a compact form due to the presence of the cubic root. However, it can be shown that it is also a quasi-homogeneous function by virtue of the algebraic conditions (60). In fact, from the above analysis and Eq. (61), it follows that if $S_{B H}$ is quasi-homogeneous, then $S_{W}$ is also a quasi-homogeneous function of the same degree. This proves that the above EMGB black hole is a quasi-homogeneous thermodynamic system with respect to both the Bekenstein-Hawking and the Wald entropy.

\section{Conclusions}

In this work, we argue that black holes are thermodynamic systems described by fundamental equations that should correspond to quasi-homogeneous functions. This means that the concept of extensivity and intensivity of black hole thermodynamic variables is not as clear and concrete as in the case of ordinary systems, which are described by homogeneous fundamental equations. Essentially, the origin of the quasi-homogeneity of black holes is already contained in the Hawking-Bekenstein entropy, which is proportional to the area and not to the volume, as in the case of ordinary systems.

From the condition of quasi-homogeneity of black holes, we derive the important property that coupling constants of gravity theories must be considered as thermodynamic variables. We prove this for the cosmological constant, the Born-Infeld parameter and the Gauss-Bonnet constant. The cosmological constant can indeed be interpreted as the cou- pling constant between the gravitational field and the matter described by the vacuum energy. In turn, the BornInfeld parameter and the Gauss-Bonnet constant are coupling constants between gravity and, respectively, the nonlinear electromagnetic field and the effective field represented by the topological term. In fact, the cosmological constant has been interpreted previously as a thermodynamic variable with properties consistent with an effective "pressure" [11]. In this context, it would be interesting to investigate the interpretation of the Born-Infeld parameter and the Gauss-Bonnet constant in the framework of black hole thermodynamics. Finally, since the explicit application of the quasi-homogeneity condition is quite simple, we can conjecture that our results hold for all the coupling constants of any generalization of Einstein gravity.

Since the degree and the coefficients of quasi-homogeneity are defined up to a multiplicative constant factor, one is tempted to use this freedom to fix the degree to 1 , by transforming the thermodynamic variables appropriately. We have shown that this procedure can lead to contradictory results. In black hole thermodynamics, the phase transition structure can be modified by the transformation of variables. The free parameter that appears in the degree of quasi-homogeneity turns out to correspond to a multiplicative constant of the metric used in GTD to describe black holes so that it does not affect the geometric properties of the equilibrium space. However, GTD is very sensitive to the transformations of variables at the level of the fundamental equation, which can completely modify the thermodynamic curvature of the system under consideration.

According to our results, quasi-homogeneity is a property of non-ordinary systems which must be handled correctly in order to avoid unphysical and contradictory results. It also leads to a deep modification of the way we interpret coupling constants in gravity theories. It would be interesting to further explore the physical consequences of these modifications.

Acknowledgements This work was carried out within the scope of the project CIAS 2312 supported by the Vicerrectoría de Investigaciones de la Universidad Militar Nueva Granada-Vigencia 2017. This work was partially supported by UNAM-DGAPA-PAPIIT, Grant no. 111617 , and by the Ministry of Education and Science of RK, Grant nos. BR05236322 and AP05133630. We thank an anonymous referee for very useful comments and suggestions.

Data Availability Statement This manuscript has no associated data or the data will not be deposited. [Authors' comment: No data is used in this work.]

Open Access This article is distributed under the terms of the Creative Commons Attribution 4.0 International License (http://creativecomm ons.org/licenses/by/4.0/), which permits unrestricted use, distribution, and reproduction in any medium, provided you give appropriate credit to the original author(s) and the source, provide a link to the Creative Commons license, and indicate if changes were made. Funded by SCOAP ${ }^{3}$. 


\section{References}

1. H.B. Callen, Thermodynamics and an Introduction to Thermostatistics (Wiley, New York, 1985)

2. H. Quevedo, M.N. Quevedo, A. Sánchez, Eur. Phys. J. C 77, 158 (2017)

3. V. Pineda, H. Quevedo, M.N. Quevedo, A. Sánchez, E. Valdés, On the physical interpretation of geometrothermodynamic metrics (2018). arXiv:1704.03071

4. H.E. Stanley, Introduction to Phase Transitions and Critical Phenomena (Oxford University Press, New York, 1971)

5. F. Belgiorno, J. Math. Phys. 44, 1089 (2003)

6. F. Belgiorno, Phys. Lett. A 312, 224 (2003)

7. F. Belgiorno, S.L. Cacciatori, Eur. Phys. J. Plus 126, 86 (2011)

8. I. Papadimitriou, K. Skenderis, JHEP 0508, 004 (2005)

9. D. Kastor, S. Ray, J. Traschen, Class. Quantum Gravity 26, 195011 (2009)

10. M. Cvetic, G.W. Gibbons, D. Kubiznak, C.N. Pope, Phys. Rev. D 84, 024037 (2011)

11. D. Kubiznak, R.B. Mann, M. Teo, Class. Quantum Gravity 34, 063001 (2017)

12. S. Amari, Differential-Geometrical Methods in Statistics (Springer, Berlin, 1985)

13. F. Weinhold, Classical and Geometrical Theory of Chemical and Phase Thermodynamics (Wiley, Hoboken, 2009)

14. G. Ruppeiner, Springer Proc. Phys. 153, 179 (2014)
15. H. Quevedo, J. Math. Phys. 48, 013506 (2007)

16. H.E. Stanley, Rev. Mod. Phys. 71, 2 (1999)

17. H. Liu, H. Lü, M. Luo, K.N. Shao, J. High Energy Phys. 1012, 054 (2010)

18. H. Quevedo, M.N. Quevedo, Electr. J. Theor. Phys. 2011, 1 (2011)

19. H. Quevedo, M.N. Quevedo, A. Sánchez, Phys. Rev. D 94, 024057 (2016)

20. H. Quevedo, M.N. Quevedo, A. Sánchez, S. Taj, Phys. Scr. 8, 084007 (2014)

21. J.E. Åman, N. Pidokrajt, Gen. Rel. Grav. 38, 1305 (2006)

22. J.E. Åman, N. Pidokrajt, Phys. Rev. D 73, 024017 (2006)

23. P.C.W. Davies, Rep. Prog. Phys. 41, 1313 (1977)

24. H. Stephani, D. Kramer, M. MacCallum, C. Hoenselaers, E. Herlt, Exact Solutions of Einstein's Field Equations (Cambridge University Press, Cambridge, 2003)

25. G.W. Gibbons, M.J. Perry, C.N. Pope, Class. Quantum Gravity 22, 1503 (2005)

26. A. Ashtekar, T. Pawlowski, C. van den Broeck, Class. Quantum Gravity 24, 625 (2007)

27. M.M. Caldarelli, G. Cognola, D. Klemm, Class. Quantum Gravity 17, $399(2000)$

28. Y.S. Myung, Y.W. Kim, Y.J. Park, Phys. Rev. D 78, 084002 (2008)

29. M. Born, L. Infeld, Proc. R. Soc. Lond. A 144, 425 (1934)

30. D.L. Wiltshire, Phys. Lett. B 169, 36 (1986)

31. D.L. Wiltshire, Phys. Rev. D 38, 2445 (1988)

32. E. Herskovich, M.G. Richarte, Phys. Lett. B 689, 192 (2010) 